\title{
PRODUCTIVIDAD DE LAS PYMES, SECTOR CAUCHO Y PLÁSTICO DE BOGOTÁ D.C. ${ }^{1}$
}

\section{José Zacarías Mayorga Sánchez ${ }^{2}$ - Jaime Augusto Porras Jiménez ${ }^{3}$}

\section{Universidad Autónoma de Colombia}

Artículo de Investigación e Innovación - Recibido: 9 de marzo de 2015 - Aceptado: 9 de septiembre de 2015

doi:

\section{RESUMEN}

Según estudios, en el sector caucho y plástico de la ciudad de Bogotá, la falta de competitividad, se deriva principalmente del bajo nivel productivo de la industria, problemas de distribución y comercialización y deficiente direccionamiento estratégico de las empresas. En tal sentido, el artículo aborda el entorno del sector, su desempeño productivo y su proyección en el mercado internacional; se incluye un análisis detallado de la muestra de empresas, sus aspectos administrativos, recursos humanos, producción, mercadeo y finanzas; por último, se aborda una modelación econométrica que mide la productividad de las empresas del sector. Los resultados muestran que se requieren nuevas estrategias para mejorar la productividad, la competitividad de los sectores y en general de la economía, para poder aprovechar las oportunidades que se presentan en el mercado.

\section{Palabras clave:}

Productividad, Pymes, Valor agregado, Factores productivos, competitividad.

JEL: D21, D23, D24,

\section{Si va a referenciar este artículo}

Mayorga, J. \& Porras, J. (2015). Productividad de las pymes, sector caucho y plástico de Bogotá D.C.. Económicas CUC, 36(2), 95-111. doi:

\footnotetext{
${ }^{1}$ Artículo de Investigación e Innovación. Investigación Fuente: Medición de la productividad a las pymes del sector industria fabricación de productos de caucho y de plástico en la ciudad de Bogotá D.C. Universidad Autónoma de Colombia - FUAC Línea de investigación - Productividad y Competitividad de las empresas colombianas (Pymes).

${ }^{2}$ Economista, Magister en Planeación Socioeconómica, Doctorante en Administración, Docente Investigador Fundación Universidad Autónoma de Colombia - FUAC. Jose.mayorga@fuac.edu.co

${ }^{3}$ Administrador de Empresas, Magister en Administración, PHD en Educación, Investigador Externo Fundación Universidad Autónoma de Colombia - FUAC. jaime.porras@fuac.edu.co
} 


\title{
SMES PRODUCTIVITY: \\ RUBBER AND PLASTIC INDUSTRIES IN BOGOTA
}

\begin{abstract}
Studies performed in the rubber and plastic industries from the city of Bogota show that the lack of competitiveness mainly derives from low productivity levels, distribution and commercialization problems, and deficient strategic direction of companies. This paper addresses this industry's environment, productive performance, and international market projections. An econometric modeling is executed to measure these companies' productivity. To develop this research, a mixed method was used so as to study quantitative and qualitative variables; specific data gathering techniques, like surveys, were applied. The data obtained was subjected to a coding, tabulation, and statistical analysis process based on the theoretical references in force. Results evidence the requirement of new strategies to enhance productivity, competitiveness, and in general terms, the economy of these industries in order to take advantage of market opportunities available. To conclude, work and other production factors or supplies are the source of wealth, hence, to achieve superior levels, more and better production factors and an efficiency increase in the processes are demanded.
\end{abstract}

\section{Key words:}

Productivity, SMEs, Aggregated Value, Productive Factors, Competitiveness 


\section{INTRODUCCIÓN}

Para que un país pueda producir a bajos costos, es necesario que exista una productividad mayor con respecto a otros países, por lo que David Ricardo en la teoría del valor y la distribución, consideró que el trabajo era uno de los pilares de la productividad, porque el valor no sólo es determinado por el trabajo que se incorpora a las herramientas, instrumentos, edificios, etc., sino también por el trabajo pasado. (Bustelo, 1994)

Para la Organización Internacional del Trabajo, (OIT), “...los productos son fabricados como resultados de la integración de cuatro elementos principales: tierra, capital, trabajo y organización. La relación de estos elementos a la producción es una medida de la productividad." (Carballal, 2005 p. 5). Así, que aunque a lo largo del tiempo ha habido diferentes discusiones, estudios y teorías sobre las variables que miden la productividad, éstas pueden incluir los anteriores cuatro elementos.

Sin lugar a dudas, la productividad debe ser vista como una medida de eficiencia en la utilización de los factores de producción y específicamente de la utilización de los recursos de que dispone una empresa en su proceso productivo, de este modo, Como lo menciona Kurosawa (1983), la productividad se puede definir, como una medida para evaluar cualquier tipo de actividad productiva de la sociedad o como un grado de significancia y efectividad de las actividades que utilizan a la naturaleza.

En la práctica, la aplicación de los conceptos teóricos han llevado a definirla como una relación simple entre producto y factores, por lo que la International Labor Office, en 1969 la llamó "Productividad Total de los Factores".

En los años recientes, la productividad ha tomado gran importancia en el desarrollo de la economía y se le atribuye la responsabilidad en la competitividad, no sólo a nivel empresarial, sino internacional, y como lo sentencia Porter (2007), en su teoría de la Ventaja Competitiva, para que un país desarrolle una ventaja de ésta índole, es necesario que alcance un liderazgo en costos. Lo anterior significa, que produce a menor costo, o dicho de otra manera mayor productividad de los factores, diferenciación y enfoque, pero según el autor es fundamental que el país defina qué tipo de ventaja quiere lograr y concentrarse en hacerlo.

\section{Concepto general del sistema de medición de la productividad por la metodología del Valor Agregado 4}

Si las empresas manifiestan que quieren mejorar su productividad, necesariamente hay que medirla. Pues, los indicadores de rentabilidad tradicionales son adecuados, pero para lograr una medición significativa, deben estar ligados con la productividad, ya que ésta, afecta en gran medida los rendimientos de la empresa. Además, las mediciones de la misma fortalecen la planeación estratégica de las organizaciones, que pueden utilizar el comportamiento de estos índices durante un tiempo determinado, como una herramienta de diagnóstico, que ayuda a identificar áreas problemáticas que requieren de una atención inmediata.

Sin lugar a dudas, la creación de valor es hoy la mayor preocupación de la gestión estratégica de los negocios, por esta razón, tener claro el concepto y medir el valor agregado de la empresa es fundamental. El valor agregado, está compuesto por: los pagos a trabajadores, depreciación, pagos al gobierno (impuestos), intereses, arrendamientos y utilidades de la empresa ${ }^{5}$. (Centro Nacional de Productividad, 2008)

\footnotetext{
4 Tomado de: "Medición de la Productividad del Valor Agregado y sus aplicaciones prácticas" por Masayasi Shimizu y otros. Traducido por José Alfredo Roa. Japan Productivity Center for Socio - Economic Development (JPC). Santiago de Cali, junio de 2001.

${ }^{5}$ El valor agregado se traduce como la creación de valor, en este proceso intervienen diferentes agentes los cuales hacen aportes y reciben retribuciones, éstas son las que más adelante van a servir para el cálculo del valor agregado.
} 


\section{METODOLOGIA}

Para el desarrollo de la investigación se abordó un modelo mixto, en el cual se estudian, variables cuantitativas y cualitativas, se emplearon técnicas específicas en la recolección de información como encuestas, y la información obtenida se sometió a un proceso de codificación, tabulación y análisis estadístico con base en los referentes teóricos actuales.

Para la recolección de la información, se clasificaron las fuentes en primarias y secundarias; esta información se obtuvo del macro-ambiente (que se abordó desde las fuentes secundarias, tales como referentes teóricos, investigaciones hechas anteriormente por universidades, y otras entidades, y bases de datos publicadas por los organismos de control, entre otros). A nivel micro o empresarial, se obtuvo de las fuentes primarias (encuestas realizadas a las empresas, bases de datos de la Cámara de Comercio de Bogotá y de la Superintendencia de Sociedades).

Para medir la productividad de las empresas del sector, se diseñó un modelo econométrico, el cual fue calibrado y se comprobó su viabilidad de aplicación.
Modelación Econométrica. En primera instancia, se tomó de la Encuesta Anual Manufacturera del DANE en los años 2000- 2011, variables como: Valor agregado, producción bruta, consumo intermedio, materias primas consumidas, personal ocupado (número), personal remunerado (cantidad), personal vinculado a la producción (número), remuneración total, sueldos y salarios, prestaciones sociales, activos fijos, energía eléctrica consumida (Millones de $\mathrm{KWH}$ ). Al analizar las variables, se encontró que las que mejor explican el nivel de productividad del valor agregado del sector caucho y plástico son: consumo intermedio (C), materias primas consumidas (MP), prestaciones sociales (PS) y Valor ventas (V). (DANE: 2012). Dado que en la investigación se trabaja con estas variables, para realizar la regresión se hizo un ajuste a las variables independientes (X1 a X4) y a la variable dependiente (Valor agregado) calculando el logaritmo natural de cada una de ellas.

Donde: $\mathrm{C}=$ Consumo intermedio; $\mathrm{MP}$ $=$ Materias primas consumidas; PS = Prestaciones sociales y $\mathrm{V}=$ Ventas

Tabla 1

Regresión lineal modelo valor agregado

\begin{tabular}{|c|c|c|c|c|}
\hline Variable & Coefficient & Std. Error & t-Statistic & Prob. \\
\hline $\mathrm{C}$ & -0.587059 & 0.522881 & -1.122741 & 0.2941 \\
\hline MP & -1.279692 & 0.483927 & -2.590855 & 0.0321 \\
\hline PS & -0.352750 & 0.297219 & -1.186834 & 0.2693 \\
\hline $\mathrm{V}$ & -2.490119 & 0.717780 & 3.469195 & 0.0085 \\
\hline R-squared & 0.993935 & Mean dependent var & & 14.58317 \\
\hline Adjusted R-squared & 0.991660 & S.D. dependent var & & 0.328699 \\
\hline S.E. of regression & 0.030017 & Akaike info criterion & & -3.912898 \\
\hline Sum squared resid & 0.007208 & Schwarz-Quinn criter. & & -3.751263 \\
\hline Log likelihood & 27.47739 & Hannan-Quinn criter. & & -3.972742 \\
\hline F-statistic & 437.0078 & Durbin-Watson stat & & 2.169999 \\
\hline Prob(F-statistic) & 0.000000 & & & \\
\hline
\end{tabular}

Fuente: Elaboración propia, presente investigación - 2014 
Al realizar la regresión, Tabla 1 , se alcanzó un coeficiente de correlación del $99.9 \%$, lo que significa que las variables independientes están fuertemente relacionadas con la variable dependiente (valor agregado). La prueba conjunta arrojó un valor $\mathrm{F}$ más alto que el valor crítico de $\mathrm{F}$, lo que demuestra que ésta es muy significativa estadísticamente, ya que el error crítico tiende a cero. Con esto se demuestra que el modelo tiene una correlación alta entre la variable dependiente y las independientes. Por su parte, en la prueba individual, las variables independientes tuvieron una probabilidad o error crítico del estadístico $\mathrm{T}$ menor al $30 \%$, lo que indica que éstas explican significativamente a la variable dependiente.

Interpretación de los coeficientes: La explicación cuantitativa del efecto que tiene un cambio en alguna de las variables independientes sobre la variable dependiente, permaneciendo el resto de variables constantes (ceteris paribus) permitió establecer: Si se incrementa en $1 \%$ el valor de las materias primas consumidas, siempre y cuando el resto de los factores se mantengan constantes, el valor agregado disminuye en $127 \%$ : $\mathrm{Si}$ se incrementa en $1 \%$ el valor de las prestaciones sociales, siempre y cuando el resto de los factores se mantengan constantes, el valor agregado disminuye en $35 \%$ y si se incrementa en $1 \%$ el valor de las ventas, siempre y cuando el resto de los factores se mantengan constantes, el valor agregado aumenta en $249 \%$. Esto muestra que la variable dependiente es elástica con respecto a los cambios en cada una de las variables independientes.

Como resultado se obtuvo el modelo:

Ecuación 1. Representación del modelo econométrico del valor agregado:
Logaritmo de VA = - $0.587059-1.279695 \operatorname{In}(M P)$

- $0.352750 \operatorname{In}(P S)+2.490119 \operatorname{In}(V)$

Donde: $\mathbf{M P}=$ Materias primas consumidas; $\mathbf{V}=$ Ventas $\mathbf{y} \mathbf{P S}=$ Prestaciones sociales pagadas.

Al aplicar el antilogaritmo a los dos lados de la igualdad, resultó la siguiente ecuación:

Ecuación 2 - modelo econométrico del valor agregado aplicando antilogaritmo:

$V A={ }_{e} 0.58759-{ }_{m p} 1.279695-{ }_{p s} 0.352750+$ ${ }_{v} 2.490119$

El modelo es idóneo para hacer el cálculo del valor agregado de las empresas productoras pertenecientes al sector caucho y plástico, debido a que, como se mencionó anteriormente, tiene una correlación del 99.9\%. Los supuestos generales del modelo lineal, son los siguientes:

- $\mathrm{E}(\mathrm{Yt} / \mathrm{Xt})=a+\mathrm{Xt} B \rightarrow \mathrm{El}$ modelo puede representarse

- $\varepsilon t \sim \mathrm{N}\left(0 ; \sigma^{\wedge} 2 . \mathrm{I}\right) \rightarrow \mathrm{El}$ error tiene una distribución Normal.

- $\rho(X)=k \rightarrow X$ es fija y de rango (Txk) completo (no perfecta multicolinealidad)

- El error presenta una matriz de varianza y covarianza:

- $\mathrm{E}\left(\varepsilon \varepsilon^{\prime}\right)=\mathrm{E}\left(\varepsilon^{\wedge} 2\right)=\operatorname{Var}(\varepsilon)$ Homoscesdasticidad.

- $\mathrm{E}(\varepsilon \mathrm{t}, \varepsilon \mathrm{s})=\operatorname{Cov}(\varepsilon t, \varepsilon \mathrm{s})=0$ no autocorrelación.

Para mostrar que el modelo es idóneo se procedió a realizar pruebas de Autocorrelación, Heterocedasticidad y Multicolinealidad, para lo cual se realizó prueba de normalidad, a través del test de normalidad Jarque-Bera, figura 1. 


$$
J B=\frac{T-k}{6}\left[S^{2}+\frac{(K-3)^{2}}{4}\right]
$$

$\rightarrow$ Si el JB es menor 5.99 no se rechaza la hipótesis nula

T: Tamaño de muestra; K: Es la kurtosis; S: Es la asimetría; k: Número de regresoras

Como se puede observar en la figura 1, el test Jarque-Bera es menor a 5.99 por lo que no se rechaza la hipótesis nula y se afirma que los errores tienen una distribución normal. Así mismo, la asimetría tiende a cero, lo que da indicios de normalidad. Finalmente, existe una probabilidad de $72 \%$ (mayor al 5\%) de no rechazar la hipótesis nula con lo que se comprueba que el modelo cumple con el supuesto de normalidad.

Prueba de multicolinealidad. Esta situación se presenta cuando existe una relación lineal entre dos o más variables explicativas del modelo. Para esta investigación se realizó la prueba de acuerdo con el análisis de la matriz de correlaciones en la Tabla 2 .
Tabla 2.

Matriz correlaciones del modelo

\begin{tabular}{|c|c|c|c|}
\hline & $\mathrm{C} 1$ & $\mathrm{C} 2$ & C3 \\
\hline & \multicolumn{3}{|c|}{ Matriz de correlaciones } \\
\hline $\mathrm{R} 1$ & 1.000000 & 0.940052 & 0.993419 \\
\hline $\mathrm{R} 2$ & 0.940052 & 1.000000 & 0.971005 \\
\hline R3 & 0.993419 & 0.971005 & 1.000000 \\
\hline
\end{tabular}

Fuente: Elaboración propia, presente investigación - 2014

Existe una fuerte correlación entre las materias primas consumidas y el valor de las ventas (ver figura 2. El determinante de esta matriz es 0.00014 , lo que significa que tiende a cero. Esto indica una posible colinealidad en el modelo.

Esta medida arrojó resultados que muestran la presencia del problema de multicolinealidad en el modelo, sin embargo cuando en la regresión del modelo inicial el coeficiente de determinación es alto (99.9\%) y los coeficientes de determinación individuales estimados son de igual manera significativos, este problema no resulta relevante, por lo que se puede hacer caso omiso de su existencia. Dado que este modelo se corrió en forma logarítmica, no es necesario hacer pruebas de Heterocedasticidad.

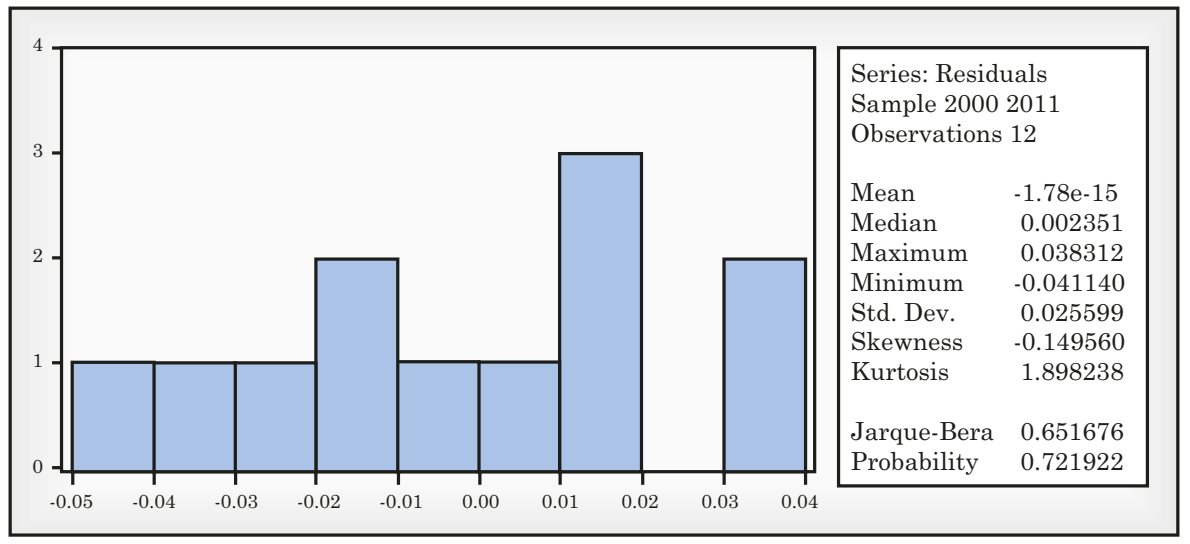

Figura 1. Test de Normalidad Jarque-Bera

Fuente: Elaboración propia, presente investigación - 2014 


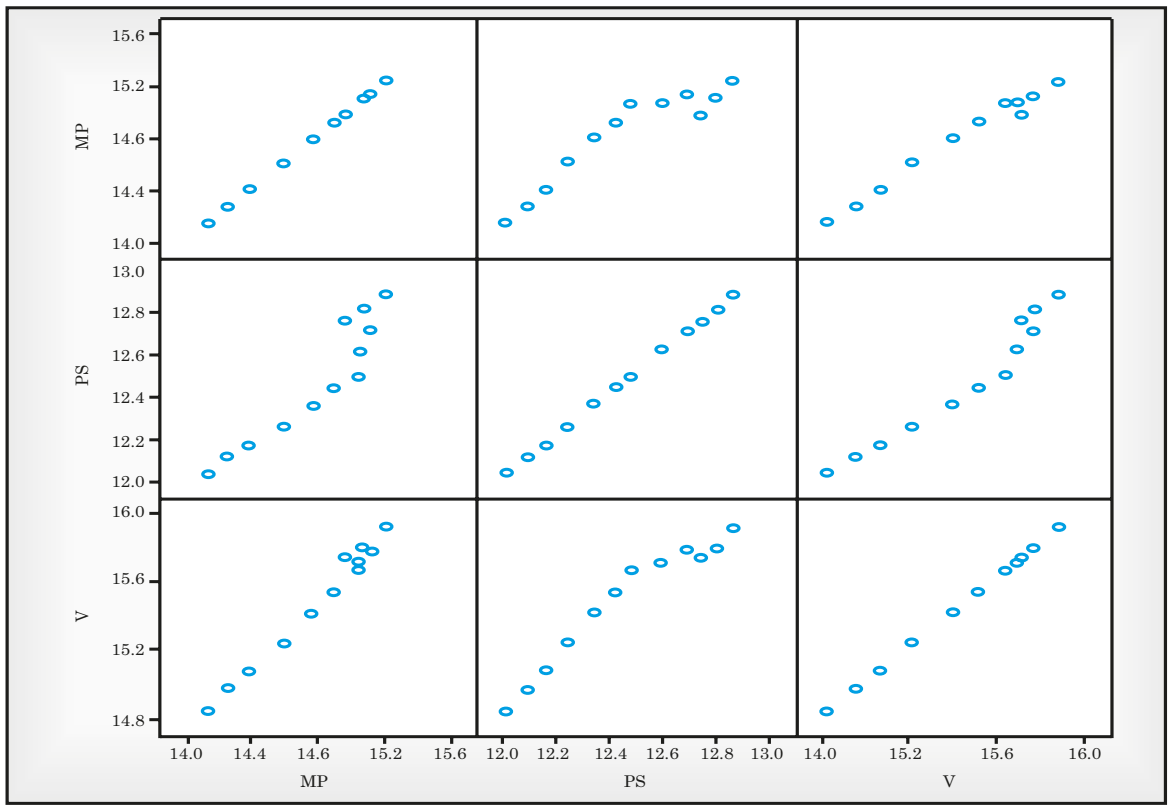

Figura 2. Gráfica matriz de correlaciones

Fuente: Elaboración propia, presente investigación - 2014.

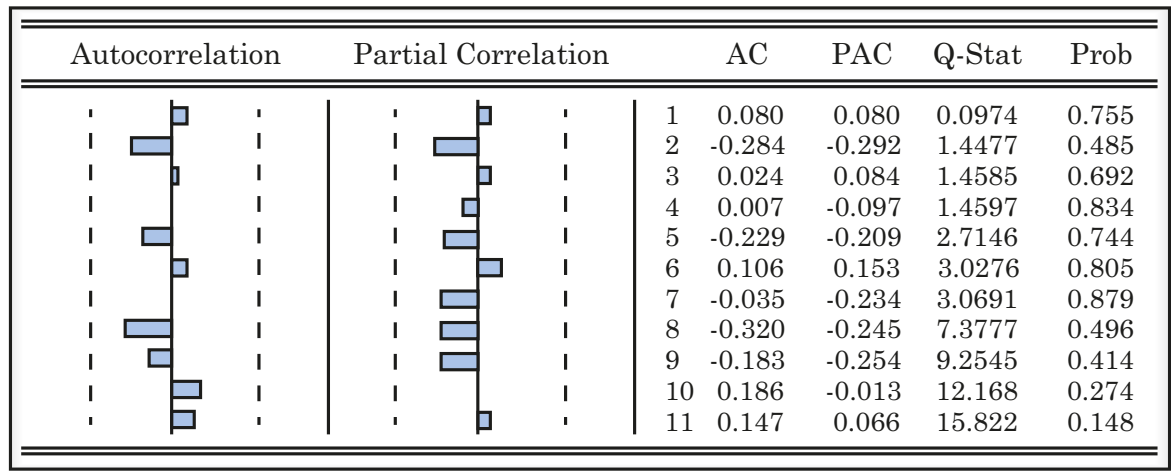

Figura 3. Correlograma

Fuente: Elaboración propia, presente investigación - 2014.

\section{Prueba de autocorrelación.}

Ecuación 3. Método de Durbin Watson

$$
d=\frac{\Sigma(e t-e t-1)^{2}}{\Sigma e t^{2}}
$$

Tras lo anterior, el estadístico DurbinWatson de este modelo, se estimó con un valor de 1.5128. Puesto que el número de observaciones es igual a 12 y el número de parámetros igual a cuatro (4), se busca en la tabla DW con un nivel de significancia del $5 \%$ y se observa que $\mathrm{dl}=0.522$ y $\mathrm{dt}=2.177$. Con esto se concluye que existen sospechas de una autocorrelación positiva. Por otra parte, como se aprecia en la figura 3 , no hay autocorrelación, debido a que los coeficientes de autocorrelación no pasan de las bandas.

Prueba de Breusch - Godfrey. Con una probabilidad de $71 \%$ (Mayor a 5\%) no se rechaza la hipótesis nula, por lo cual el modelo no presenta autocorrelación. 
Tabla 3.

Prueba de Breusch - Godfrey

\begin{tabular}{|c|c|c|c|c|}
\hline \multicolumn{5}{|c|}{ Breusch-Godfrey Serial Correlation LM Test } \\
\hline F-statisc & 0.076946 & Prob. $F(1,7)$ & & 0.7895 \\
\hline Obs*R-squared & 0.130473 & Prob. Chi-Square(1) & & 0.7179 \\
\hline \multicolumn{5}{|c|}{$\begin{array}{l}\text { Test Equation: } \\
\text { Dependent Variable:RESID } \\
\text { Method: Least Squares }\end{array}$} \\
\hline \multicolumn{5}{|c|}{$\begin{array}{l}\text { Sample: } 20002011 \\
\text { Included observations: } 12 \\
\text { Presample missing value lagged residuals sett to zero }\end{array}$} \\
\hline Variable & Coefficient & Std. Error & t-Statistic & Prob. \\
\hline $\mathrm{C}$ & 0.018247 & 0.559814 & 0.032594 & 0.9749 \\
\hline MP & 0.000553 & 0.525155 & 0.001054 & 0.9992 \\
\hline PS & 0.018847 & 0.323231 & 0.058308 & 0.9551 \\
\hline $\mathrm{V}$ & -0.016932 & 0.765593 & -0.022116 & 0.9830 \\
\hline RESID (-1) & 0.136128 & 0.490745 & 0.277392 & 0.7895 \\
\hline$R$-squared & 0.010873 & Mean dependent var & & $4.15 \mathrm{E}-15$ \\
\hline Adjusted R-squared & -0.554343 & S.D. dependent var & & 0.025599 \\
\hline S.E. of regression & 0.031915 & Akaike info criterion & & -3.757164 \\
\hline Sum squared resid & 0.007130 & Schwarz-Quinn criter. & & -3.555120 \\
\hline Log likelihood & 27.54298 & Hannan-Quinn criter. & & -3.8311968 \\
\hline F-statistic & 0.019237 & Durbin-Watson stat & & 1.605204 \\
\hline $\operatorname{Prob}($ F-statistic) & 0.999086 & & & \\
\hline
\end{tabular}

Fuente: Elaboración propia, presente investigación - 2014.

En conclusión, se puede decir que este modelo no presentó ninguno de los problemas más comunes de la regresión lineal, lo que demuestra y garantiza que, según la investigación hecha por Gaviria \& Sierra (2012), los coeficientes estimados son insesgados, tienen variación mínima, son consistentes y tienen una distribución normal.

\section{RESULTADOS}

\section{Desempeño del sector}

Para el Departamento Administrativo Nacional de Estadística - DANE (2012), el sector caucho y plástico, presentó un crecimiento casi constante desde el año 2001 al 2006, sin embargo a partir del año 2008 se desaceleró su producción (Ver Figura 4), debido principalmente a que en este año, Estados Unidos entró en crisis económica, la cual afectó la industria manufacturera mundial. En el caso colombiano, se vio reflejada en la producción del año 2009, que tuvo una variación porcentual de $-3 \%$ con respecto al año 2008 (DANE, 2012). Este efecto se debe a que este sector está muy relacionado con las industrias de alimentos, bebidas y productos químicos.

Con respecto al crecimiento del sector, si se le relaciona con el de la industria manufacturera y con el crecimiento del PIB nacional, (ver Figura 5), el sector Caucho y Plástico, a comienzo del presente siglo, tiene un crecimiento similar al del PIB de la industria manufacturera y al PIB na- 
Producción bruta Caucho y plástico

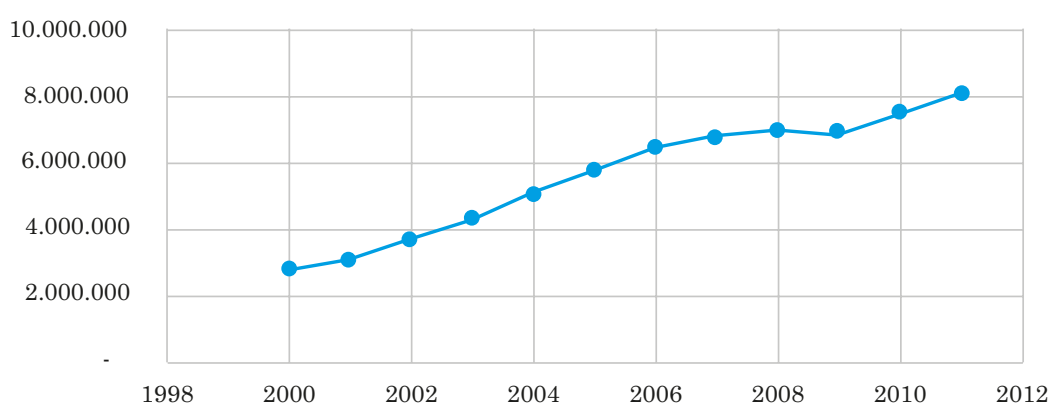

Figura 4. Producción bruta Caucho y Plástico, 2000 - 2012

Fuente: Elaboración propia con base en datos tomados del DANE, 2012

Crecimiento económico (PIB Caucho y plástico. PIB Manufactura y PIB Nacional)

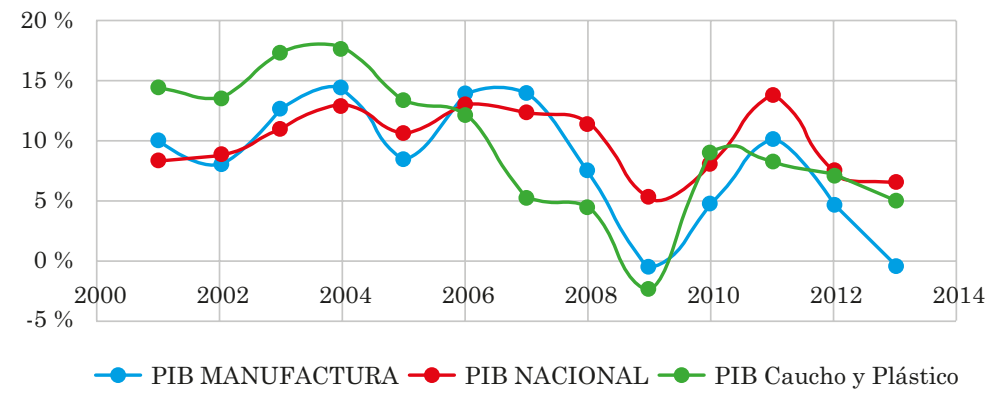

Figura 5. Crecimiento económico (PIB Manufactura - PIB Nacional- PIB caucho y plástico), 2002 -2014

Fuente: Elaboración propia con datos tomados del Banco de la República, 2014

cional $^{6}$. A partir del año 2007, su desempeño fue fuertemente afectado por la crisis económica y llegó a su nivel más bajo en el año 2009.

Es importante resaltar el impacto que el comportamiento del sector tiene en la economía nacional, pues como lo menciona el Dr. Carlos Salamanca, presidente de la Asociación Colombiana de Industrias Plásticas, Acoplásticos (2014), el sector agrupa aproximadamente 600 empresas y genera más de 40.000 empleos directos.

${ }^{6}$ Esta similitud de comportamiento se evidencia en el coeficiente de correlación de estas variables, el cual es superior al $80 \%$, según cálculo hecho con base en datos reportados por el Banco de la República.

\section{Productividad laboral, sector caucho y plástico.}

Para calcular este indicador se tomó como base la relación entre la producción bruta y el número de trabajadores por la industria durante la última década. En el marco de la globalización, la productividad laboral representa un mecanismo predominante para alcanzar la competitividad internacional, sin embargo, según Garay (1998), ésta apoyará a la competitividad siempre y cuando provenga de mejoras en los procesos productivos y en los indicadores de gestión de los trabajadores o de la introducción de innovaciones en el proceso productivo. Ver figura 6. 
Productividad laboral

(Miles de millones de pesos)

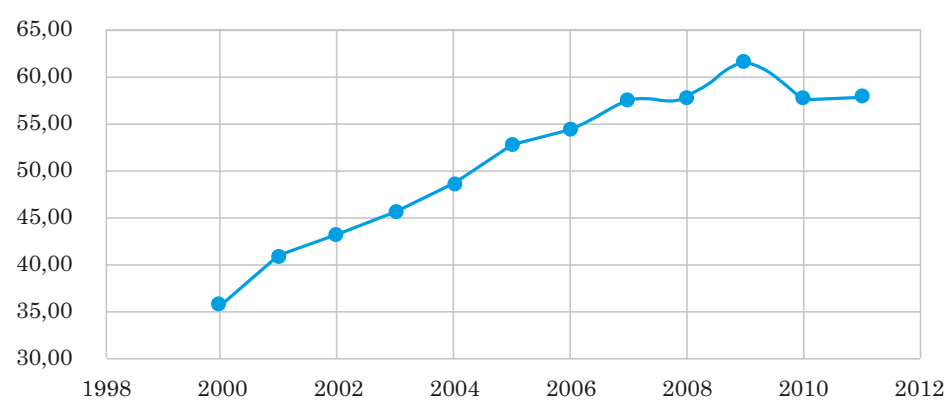

Figura 6. Productividad laboral sector Caucho y Plástico

Fuente: Elaboración propia - DANE, 2012

Productividad del costo de capital

(Miles de millones de pesos)

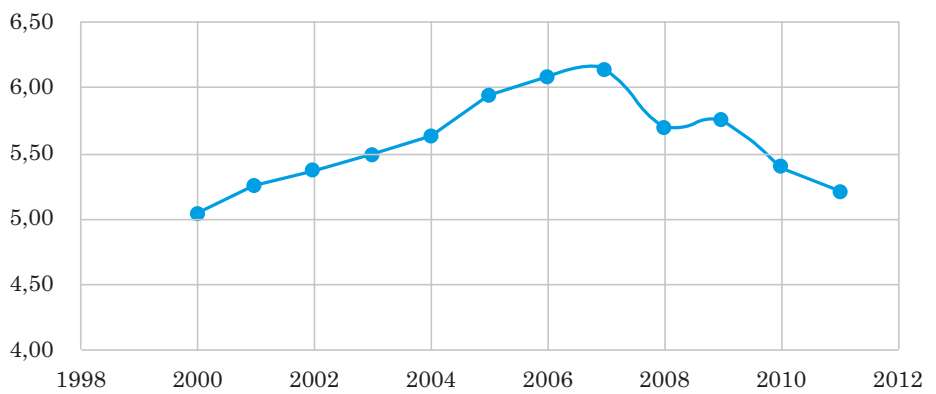

Figura 7. Productividad del Capital del sector Caucho y Plástico

Fuente: Elaboración propia - DANE, 2012

\section{Productividad del costo laboral.}

Este indicador se mide como la relación entre el valor agregado por cada peso pagado en sueldos y salarios. Un valor elevado de este indicador muestra que la industria tiene ventajas competitivas frente a otros países. Para la industria del sector Caucho y Plástico, este indicador no presenta buenos resultados ya que en la última década tuvo un crecimiento de tan sólo el 4\%, lo que quiere decir que el sector no es competitivo y puede indicar que el nivel tecnológico de la industria no es el adecuado, por lo tanto, la productividad del trabajo es baja. Ver figura 7.

\section{Nivel salarial.}

El nivel salarial es la relación entre el costo de personal y el número de empleados. Como se aprecia en la figura 8, su crecimiento ha sido constante, aunque entre el año 2009 y 2010 permaneció casi estático debido a la crisis económica vivida en esa época.

\section{Caracterización de las empresas}

A continuación se presentan los resultados obtenidos de la encuesta aplicada a una muestra de empresas seleccionadas aleatoriamente. Los resultados permiten 
Nivel salarial sector Caucho y Plástico

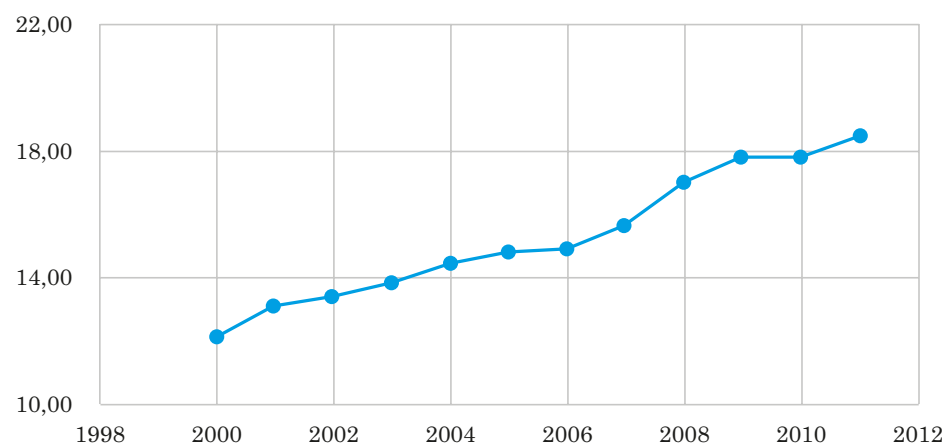

Figura 8. Nivel salarial del sector Caucho y Plástico

Fuente: Elaboración propia - DANE, 2012

\section{Gestión administrativa y de talento humano}

Tabla 4.

Indicadores de Gestión de Talento Humano.

\begin{tabular}{lcccc}
\hline \multicolumn{1}{c}{ Nit } & $\begin{array}{c}\text { \% Ausentismo } \\
\text { Anual }\end{array}$ & $\begin{array}{c}\text { \% Rotación de } \\
\text { Personal }\end{array}$ & $\begin{array}{c}\text { \% Accidentalidad } \\
\text { Laboral }\end{array}$ & $\begin{array}{c}\text { \% Capacitaciones } \\
\text { Anual }\end{array}$ \\
\hline Empresa 1 & $2 \%$ & $20 \%$ & $2 \%$ & $60 \%$ \\
\hline Empresa 2 & $3 \%$ & $10 \%$ & $2 \%$ & $5 \%$ \\
\hline Empresa 3 & $2 \%$ & $11 \%$ & $3 \%$ & $15 \%$ \\
\hline Empresa 4 & $3 \%$ & $12 \%$ & $4 \%$ & $8 \%$ \\
\hline Empresa 5 & $0 \%$ & $0 \%$ & $3 \%$ & $50 \%$ \\
\hline Empresa 6 & $3 \%$ & $10 \%$ & $3 \%$ & $50 \%$ \\
\hline Empresa 7 & $5 \%$ & $5 \%$ & $0 \%$ & $50 \%$ \\
\hline Empresa 8 & $0 \%$ & $0 \%$ & $\mathbf{2 , 0} \%$ & $6 \%$ \\
\hline Promedio & $\mathbf{3 , 0} \%$ & $\mathbf{1 0 , 0} \%$ & $\mathbf{3 4 , 0} \%$ \\
\hline
\end{tabular}

Fuente: Elaboración propia, presente investigación - 2014

establecer cómo es su situación financiera, marketing, administración, productividad, entre otras.

El $100 \%$ de las empresas tiene definida su misión, visión, principios, valores y plan estratégico vigente. El 75\% de las empresas realiza inversión en investigación y desarrollo y un $88 \%$ invierte mensualmente en capacitación del personal.

El porcentaje promedio de ausentismo anual es de 3\%, (Tabla 4), y no genera retrasos o pérdidas en la producción de la empresa; la rotación de personal es en promedio del $10 \%$, porcentaje que debe ser considerado por la administración, debido a que representa pérdidas para la empresa por el costo en capacitación, afiliaciones y otros gastos derivados de la contratación, y de los efectos en la productividad laboral. El índice de accidentabilidad es del $2 \%$ relativamente bajo; las compañías realizan capacitación de su personal, lo cual es un buen indicador para la productividad de las mismas. Por otra parte, el $75 \%$ de las empresas estudiadas, cuentan con programas de responsabilidad social empresarial. 
Como se aprecia en la Tabla 5, la mayoría de los empleados se ubica en la escala técnico y/o tecnólogo, con un buen nivel de participación de formación profesional.

Tabla 5.

Nivel educativo empleados

\begin{tabular}{|c|c|c|c|c|c|c|}
\hline Nit & $\begin{array}{c}\text { Total } \\
\text { Empleados }\end{array}$ & Primaria & Bachiller & $\begin{array}{l}\text { Técnico y/o } \\
\text { Tecnólogo }\end{array}$ & Profesional & Posgrado \\
\hline Empresa 1 & 50 & $0 \%$ & $0 \%$ & $60 \%$ & $30 \%$ & $10 \%$ \\
\hline Empresa 2 & 37 & $0 \%$ & $0 \%$ & $27 \%$ & $54 \%$ & $19 \%$ \\
\hline Empresa 3 & 35 & $0 \%$ & $0 \%$ & $29 \%$ & $57 \%$ & $14 \%$ \\
\hline Empresa 4 & 8 & $0 \%$ & $0 \%$ & $25 \%$ & $50 \%$ & $25 \%$ \\
\hline Empresa 5 & 120 & $0 \%$ & $0 \%$ & $36 \%$ & $17 \%$ & $13 \%$ \\
\hline Empresa 6 & 30 & $0 \%$ & $0 \%$ & $67 \%$ & $33 \%$ & $10 \%$ \\
\hline Empresa 7 & 26 & $0 \%$ & $0 \%$ & $38 \%$ & $31 \%$ & $12 \%$ \\
\hline Empresa 8 & 21 & $0 \%$ & $0 \%$ & $50 \%$ & $35 \%$ & $15 \%$ \\
\hline \multicolumn{2}{|c|}{ Promedio } & $0,0 \%$ & $0,0 \%$ & $41 \%$ & $38 \%$ & $15 \%$ \\
\hline
\end{tabular}

Fuente: Elaboración propia, presente investigación - 2014 .

Tabla 6.

Procesos en las empresas analizadas

\begin{tabular}{cccccc}
\hline Nit & $\begin{array}{c}\text { \% cumplimiento } \\
\text { de pedidos: }\end{array}$ & \% devoluciones & $\begin{array}{c}\text { \% cumplimiento } \\
\text { producción } \\
\text { planeada }\end{array}$ & $\begin{array}{c}\text { Utilización } \\
\text { capacidad } \\
\text { instalada }\end{array}$ & $\begin{array}{c}\text { \% tiempo } \\
\text { mantenimiento }\end{array}$ \\
\hline Empresa 1 & $95 \%$ & $1 \%$ & $95 \%$ & $90 \%$ & $50 \%$ \\
\hline Empresa 2 & $100 \%$ & $5 \%$ & $87 \%$ & $77 \%$ & $20 \%$ \\
\hline Empresa 3 & $100 \%$ & $7 \%$ & $96 \%$ & $67 \%$ & $50 \%$ \\
\hline Empresa 4 & $100 \%$ & $12 \%$ & $87 \%$ & $56 \%$ & $15 \%$ \\
\hline Empresa 5 & $100 \%$ & $5 \%$ & $100 \%$ & $80 \%$ & $60 \%$ \\
\hline Empresa 6 & $95 \%$ & $3 \%$ & $95 \%$ & $85 \%$ & $40 \%$ \\
\hline Empresa 7 & $87 \%$ & $11 \%$ & $83 \%$ & $50 \%$ & $30 \%$ \\
\hline Empresa 8 & $90 \%$ & $3 \%$ & $90 \%$ & $90 \%$ & $50 \%$ \\
\hline Promedio & $\mathbf{9 6 , 0} \%$ & $\mathbf{6 , 0} \%$ & $\mathbf{9 2 , 0} \%$ & $\mathbf{7 4 , 0} \%$ & $\mathbf{3 9 , 0} \%$ \\
\hline
\end{tabular}

Fuente: Elaboración propia, presente investigación - 2014.

Tabla 7.

Controles y accesibilidad a la tecnología en la gestión de procesos

\begin{tabular}{ccccc}
\hline $\begin{array}{c}\text { La empresa } \\
\text { cuenta con: }\end{array}$ & $\begin{array}{c}\text { Control } \\
\text { ambiental }\end{array}$ & $\begin{array}{c}\text { Automatización de } \\
\text { procesos }\end{array}$ & $\begin{array}{c}\text { Control de } \\
\text { calidad }\end{array}$ & $\begin{array}{c}\text { Tecnología de } \\
\text { punta }\end{array}$ \\
\hline $1 . \mathrm{No}$ & $13 \%$ & $0 \%$ & $0 \%$ & $0 \%$ \\
\hline $2 . \mathrm{Si}$ & $87 \%$ & $100 \%$ & $100 \%$ & $100 \%$ \\
\hline
\end{tabular}

Fuente: Elaboración propia, presente investigación - 2014. 
Tabla 8.

Nivel de endeudamiento

\begin{tabular}{cccc}
\hline Nit & $\begin{array}{c}\text { Endeudamiento } \\
\text { Total }\end{array}$ & $\begin{array}{c}\text { Endeudamiento } \\
\text { Corto Plazo }\end{array}$ & $\begin{array}{c}\text { Endeudamiento } \\
\text { Largo Plazo }\end{array}$ \\
\hline Empresa 1 & $93,0 \%$ & $93,0 \%$ & - \\
\hline Empresa 2 & $90,0 \%$ & $90,0 \%$ & - \\
\hline Empresa 3 & $203,0 \%$ & $85,0 \%$ & $118,0 \%$ \\
\hline Empresa 4 & $9,0 \%$ & $8,0 \%$ & $1,0 \%$ \\
\hline Empresa 5 & $318,0 \%$ & $220,0 \%$ & $98,0 \%$ \\
\hline Empresa 6 & $102,0 \%$ & $38,0 \%$ & $64,0 \%$ \\
\hline Empresa 7 & $188,0 \%$ & $47,0 \%$ & $141,0 \%$ \\
\hline Empresa 8 & $90,0 \%$ & $90,0 \%$ & $\mathbf{8 4} \%$ \\
\hline Promedio & $\mathbf{1 3 7} \%$ & $\mathbf{8 4} \%$ & \\
\hline
\end{tabular}

Fuente: Elaboración propia, presente investigación - 2014 .

Los resultados evidencian un nivel educativo, que lastimosamente no se refleja en el nivel de productividad laboral del sector.

\section{Gestión de procesos}

El cumplimiento de pedidos es de $96 \%$ y las devoluciones son del 6\%. Por otra parte, el cumplimiento de la producción planeada es del $92 \%$ y la utilización de la capacidad instalada del $74 \%$, con un promedio de tiempo de mantenimiento de un 39\% (Ver tabla 6); aspectos que en conjunto reflejan un comportamiento aceptable en la gestión de procesos, pero que debe ser evaluado y mejorado a corto plazo.

Para las empresas de este sector, es muy importante la accesibilidad a maquinaria y equipos de alta tecnología para poder optimizar la gestión de procesos y mejorar el nivel de productividad. Según la encuesta realizada, en el 100\%, el diseño es asistido por computadoras, así mismo, el 100\% tiene automatizados sus procesos y cuentan con tecnología de punta, el 100\% realiza controles de calidad y el $87 \%$ ejecuta control ambiental en su proceso de producción. (Ver Tabla 7)

\section{Gestión de mercadeo}

Según los datos recolectados, el $88 \%$ de las empresas exporta, por otra parte, en promedio, el $37 \%$ vende productos de temporada.

\section{Gestión financiera}

El 75\% de las empresas, pertenece al Régimen Común y el 25\% son Grandes Contribuyentes; la principal fuente de financiación de éstas son los recursos propios, seguida del crédito comercial. Se resalta que el $78 \%$ de sus ventas, en promedio, son de contado.

Es preocupante que el endeudamiento total de estas empresas sea tan alto, (ver tabla 8), y que en promedio, a corto plazo, sea del $84 \%$, muy por encima del endeudamiento del total de las empresas a nivel nacional, el cual es del 52\%. Un nivel de endeudamiento alto es conveniente solamente cuando la tasa de rendimiento del activo es más alta que el costo promedio del capital. Para el caso en estudio, es evidente que hay un serio problema en lo concerniente al manejo financiero de las empresas. 
Los resultados incluidos en la tabla 9, muestran que el margen bruto de rentabilidad promedio es del 70\%, nivel alto en comparación con la rentabilidad bruta de las empresas nacionales de este sector, que es del $22 \%$, sin embargo, este nivel contrasta con el margen neto que apenas llega al 8\%

En el análisis se aprecia que, el incremento del activo promedio fue de $8 \%$, sin embargo este dato está sesgado por una de las empresas que tuvo un nivel negativo de crecimiento. Si se realiza el cálculo promedio, sin tomar en cuenta esta empresa, el crecimiento de los activos para el año 2013 es en promedio del 19\%, alto en comparación con aquellas pertenecientes a este sector a nivel nacional, las cuales tuvieron un crecimiento negativo del $-15 \%$.

Finalmente se pudo establecer que el $13 \%$ de las empresas cotiza en bolsa, también, que el $37 \%$ ofrece incentivos financieros a sus clientes por pronto pago.

Los indicadores del sector, incluidos en la tabla 10 , muestran un panorama com- plejo para éstas empresas, aspecto que se hace evidente en el comportamiento de las incluidas en el estudio.

\section{Medición de la productividad utilizando la metodología del valor agregado}

Con base en el modelo econométrico elaborado y la información recolectada por medio de la encuesta realizada a empresas pertenecientes al sector industria caucho y plástico, se hizo la medición de la productividad, utilizando la metodología del valor agregado, siendo los resultados los reflejados en la tabla 11.

El valor agregado promedio es de $\$ 11.490 .125$ (valor representado en miles de pesos) en el año 2013, que equivale al $15 \%$ comparado con el valor agregado del sector manufacturero en el 2012 y el $2 \%$ comparado con el valor agregado nacional en este mismo año. De igual manera el promedio del valor agregado de estas cinco empresas, comparados con el valor agregado de la Ciudad de Bogotá en el 2012, representa un 7\% (DANE, 2013).

Tabla 9 .

Rentabilidad y crecimiento de las empresas estudiadas

\begin{tabular}{|c|c|c|c|c|c|c|c|}
\hline $\begin{array}{c}\text { Empresas } \\
\text { Nit }\end{array}$ & $\begin{array}{c}\text { Margen } \\
\text { Bruto de } \\
\text { Rentabilidad }\end{array}$ & $\begin{array}{c}\text { Margen } \\
\text { Operacional } \\
\text { de } \\
\text { Rentabilidad }\end{array}$ & $\begin{array}{c}\text { Margen } \\
\text { Neto de } \\
\text { Rentabilidad }\end{array}$ & $\begin{array}{c}\text { Rentabilidad } \\
\text { del } \\
\text { patrimonio }\end{array}$ & $\begin{array}{c}\text { Rentabilidad } \\
\text { del Activo }\end{array}$ & $\begin{array}{c}\text { Incremento } \\
\text { del Activo }\end{array}$ & $\begin{array}{c}\text { Incremento } \\
\text { del } \\
\text { Patrimonio }\end{array}$ \\
\hline Empresa 1 & $33 \%$ & $56 \%$ & $12 \%$ & $7 \%$ & $188 \%$ & $17 \%$ & $13 \%$ \\
\hline Empresa 2 & $74 \%$ & $5 \%$ & $1 \%$ & $7 \%$ & $1 \%$ & $11 \%$ & $12 \%$ \\
\hline Empresa 3 & $86 \%$ & $20 \%$ & $5 \%$ & $45 \%$ & $6 \%$ & $5 \%$ & $12 \%$ \\
\hline Empresa 4 & $98 \%$ & $37 \%$ & $36 \%$ & $32 \%$ & $45 \%$ & $11 \%$ & $28 \%$ \\
\hline Empresa 5 & $87 \%$ & $10 \%$ & $2 \%$ & $53 \%$ & $2 \%$ & $17 \%$ & $16 \%$ \\
\hline Empresa 6 & $85 \%$ & $13 \%$ & $7 \%$ & $32 \%$ & $6 \%$ & $71 \%$ & $19 \%$ \\
\hline Empresa 7 & $54 \%$ & $0 \%$ & $0 \%$ & $0 \%$ & $0 \%$ & $5 \%$ & $5 \%$ \\
\hline Empresa 8 & $40 \%$ & $14 \%$ & $3 \%$ & $7 \%$ & $3 \%$ & $-71 \%$ & $2 \%$ \\
\hline Promedio & $70 \%$ & $19 \%$ & $8 \%$ & $23 \%$ & $31 \%$ & $8 \%$ & $13 \%$ \\
\hline
\end{tabular}

Fuente: Elaboración propia, presente investigación - 2014 . 
Tabla 10.

Indicadores empresas sector caucho y plástico a nivel nacional, 2013

\begin{tabular}{lllc}
\hline \multicolumn{1}{c}{ Indicador } & $\mathbf{2 0 1 3}$ & \multicolumn{1}{c}{ Indicador } & $\mathbf{2 0 1 3}$ \\
\hline P\&G & $1,226,454$ & Endeudamiento & $51.98 \%$ \\
\hline Ventas & $-8.35 \%$ & Endeudamiento & $108.27 \%$ \\
\hline Crecimiento en Ventas & $-225,297$ & Apalancamiento & $47.02 \%$ \\
\hline Utilidad & $-626.84 \%$ & Pasivo Total/Ventas & $78.17 \%$ \\
\hline Crecimiento en Utilidades & $-18.36 \%$ & Pasivo Corriente/Pasivo Total & 62 \\
\hline Rentabilidad Neta & $22.17 \%$ & Eficiencia & 74 \\
\hline Rentabilidad Bruta & $3.29 \%$ & Rotación de Cartera & 42 \\
\hline Rentabilidad Operativa & & Rotación de Inventarios & 94 \\
\hline Balance & $1,109,460$ & Rotación de Proveedores & \\
\hline Activos & $-15.39 \%$ & Ciclo Operativo & \\
\hline Crecimiento en Activos & $-20.30 \%$ & Liquidez & 1.34 \\
\hline Rentabilidad sobre Activos & 532,68 & Razón Corriente & 0.91 \\
\hline Patrimonio & $-22.53 \%$ & Prueba Acida & $155.152,11$ \\
\hline Crecimiento del Patrimonio & $-42.29 \%$ & & Capital de Trabajo \\
\hline Rentabilidad sobre Patrimonio & & & \\
\hline
\end{tabular}

Fuente: Gestor 2014

Tabla 11.

Valor agregado e indicadores de productividad del valor agregado de las empresas estudiadas

\begin{tabular}{lccccccc}
\hline $\begin{array}{c}\text { Empresas } \\
\text { Nit }\end{array}$ & $\begin{array}{c}\text { Valor } \\
\text { Agregado }\end{array}$ & $\begin{array}{c}\text { Nivel } \\
\text { Salarial }\end{array}$ & $\begin{array}{c}\text { Productividad } \\
\text { Laboral }\end{array}$ & $\begin{array}{c}\text { Participación } \\
\text { del trabajo }\end{array}$ & $\begin{array}{c}\text { Producción } \\
\text { Media } \\
\text { por hora/ } \\
\text { hombre }\end{array}$ & $\begin{array}{c}\text { Costo } \\
\text { laboral } \\
\text { Unitario }\end{array}$ & $\begin{array}{c}\text { Intensidad } \\
\text { del Capital }\end{array}$ \\
\hline Empresa 1 & 37.314 .522 & 7616 & 310954 & $2 \%$ & 108 & $0,02 \%$ & 17580 \\
\hline Empresa 2 & 18.179 .337 & 15708 & 227417 & $1 \%$ & 789 & $0,01 \%$ & 827472 \\
\hline Empresa 3 & 12.187 .200 & 3917 & 348206 & $1 \%$ & 121 & $0,01 \%$ & 17393 \\
\hline Empresa 4 & 6.748 .243 & 2470 & 182385 & $1 \%$ & 63 & $0,01 \%$ & 27686 \\
\hline Empresa 5 & 6.648 .055 & 14174 & 255694 & $6 \%$ & 89 & $0,06 \%$ & 28101 \\
\hline Empresa 6 & 5.232 .110 & 47790 & 174404 & $27 \%$ & 61 & $0,27 \%$ & 17706 \\
\hline Empresa 7 & 3.001 .180 & 5024 & 142913 & $4 \%$ & 50 & $0,04 \%$ & 48779 \\
\hline Empresa 8 & 2.610 .349 & 11424 & 52217 & $22 \%$ & 18 & $0,22 \%$ & -6116 \\
\hline Promedio & $\mathbf{1 1 . 4 9 0 . 1 2 5}$ & $\mathbf{1 3 . 5 1 5}$ & $\mathbf{4 6 7 . 3 9 9}$ & $\mathbf{8} \%$ & $\mathbf{1 6 2}$ & $\mathbf{0 , 0 8 \%}$ & $\mathbf{1 2 2 . 3 2 5}$ \\
\hline
\end{tabular}

Fuente: Elaboración propia, presente investigación - 2014.

Lo anterior quiere decir que aunque las empresas escogidas tienen diferentes niveles de valor agregado, en promedio se puede considerar que éste es bajo en comparación con el total de las empresas del resto del país y del sector. 
Análisis de los indicadores

\section{de productividad del valor agregado}

El promedio de las empresas tiene un nivel salarial anual de $\$ 13.515$ (miles de pesos); es necesario recordar que este indicador crece en la medida en que se aumenta la productividad laboral, que para estas empresas es de 467,398 (miles de pesos) en promedio y la participación del trabajo, que es de solamente el 8\%. Por esto, cada empresa debe tener una estrategia balanceada para saber si el nivel salarial está de acuerdo con los aportes que hacen los trabajadores al proceso productivo. En este caso, la participación del trabajo es demasiado baja, debido a que este indicador es la relación entre los pagos a trabajadores y las ganancias de la productividad y esta segunda cifra es demasiado baja en la mayoría de las empresas que mantienen un bajo margen de rentabilidad.

Según el Centro Nacional de Productividad (2008), habitualmente este indicador es bajo en las empresas que están empezando y/o en economías en desarrollo como el caso de Colombia. Esto representa una oportunidad de inversiones en bienes de capital como equipo y nuevas tecnologías para abrirse a nuevos mercados o crecer el mercado actual.

Con respecto a la producción media por hora/hombre, en promedio, estas empresas arrojaron un valor de $\$ 162$ (miles de pesos). Los empleados están constituidos en su mayoría por personas con un nivel educativo técnico o tecnólogo, esto puede ser la causa por la que estas empresas tienen un buen nivel de producción por hora/hombre, ya que se encuentran especializados en sus tareas productivas y esto hace que sean más eficientes y productivos.
Así mismo, el costo laboral unitario en promedio es de $\$ 0.08$, lo cual es muy bajo si se tiene en cuenta que este índice refleja la relación entre las remuneraciones medias y la productividad laboral. Lo ideal es que ésta crezca y sea mayor que las remuneraciones medias pagadas a los trabajadores.

Finalmente, la intensidad del capital promedio para las empresas encuestadas es de $\$ 122,325$ (miles de pesos); este indicador es la relación entre el capital operativo y el número de empleados. Este nivel es alto y tiene relación con el índice de productividad de capital, lo que refleja que estas empresas tiene una buena inversión.

\section{CONCLUSIONES}

El estudio arrojó resultados que en buena parte se explican por problemas en capacitación, que dejan entrever que el modelo de educación, la formación de emprendedores, la cultura empresarial, la cultura organizacional entre otros, no corresponden con las necesidades actuales de la economía colombiana en un contexto global. A esto se suman, problemas de tramitología, dificultades por la carencia de suficientes fuentes de financiación, altos costos de producción, incertidumbre, pocos avances en investigación y desarrollo; que se traducen en baja competitividad empresarial del país en general.

El estudio permitió visualizar los factores que han incidido en el mejoramiento de la productividad de las empresas en Bogotá y Colombia, entre ellos se pueden mencionar la liberalización y la intensificación del comercio. Esto trajo consigo la eliminación de barreras al comercio, lo cual aumentó la presión competitiva e indujo a las empresas a adoptar procesos de 
innovación tecnológica. Por otra parte, la importación de maquinaria y equipo y de bienes intermedios de alta calidad conduce a las firmas a adoptar nuevos métodos de producción y a aumentar la eficiencia. Desafortunadamente las PYME no han podido hacer uso de estos beneficios por las limitaciones especialmente de capital.

Otros factores que vienen incidiendo en la productividad en Colombia tienen que ver con la difusión del conocimiento y el tamaño de los mercados. Es evidente el poco nivel de innovación, especialmente en las pequeñas empresas y sólo es posible para aquellas que cuentan con altos niveles de inversión y que además poseen una alta relación capital-trabajo, con bajos índices de deuda.

El trabajo y los demás factores o insumos de producción son la fuente de la riqueza y por consiguiente, para lograr mayores niveles, se requiere de la utilización de más y mejores elementos de producción y el incremento de la eficiencia con la cual se utilizan.

\section{REFERENCIAS}

Gaviria, M. \& Sierra, H. (1998). Medición de la productividad en la industria manufacturera de Risaralda. Revista Paginas. 53.

Bustelo, F. (1994). Historia económica. Madrid : Complutense.

Carballal, E. (2005). Conceptos modernos de productividad. Recuperado de: http://www.oocities.org/eureka/office/4595/cmproductiv.html
Centro Nacional de Productividad (2008). Medición de la productividad del valor agregado. Técnica Administrativa, 7(2). Recuperado de: http://www.cyta. com.ar/ta0702/v7n2a3.htm

Garay, L. J. (1998). Colombia: estructura industrial e internacionalización 1967 - 1996. Recuperado de: http:// www.banrepcultural.org/blaavirtual/ economia/industrilatina/211.htm

Acoplásticos, (2014). Perfil y evolución sectorial en Colombia. Recuperado de: http://www.acoplasticos.org/index. php/actividades/ae-1acs

Kurosawa, K. (1983). Medición y análisis de la productividad a nivel de la empresa. Caracas: yrigoy.

Porter, M. (2007). Ventaja competitiva. Recuperado de: http://www.itson.mx/ micrositios/pimpiie/Documents/ventaja\%20competitiva.pdf

Departamento Administrativo Nacional de Estadística - DANE. (2012). Encuesta Integrada de Hogares. Recuperado de: https://www.dane.gov.co/ index.php/mercado-laboral/empleo-ydesempleo

Departamento Administrativo Nacional de Estadística - DANE (2013). Encuesta Anual Manufacturera. Recuperado de: https://www.dane.gov.co/ index.php/industria/encuesta-anualmanufacturera-eam

Departamento Administrativo Nacional de Estadística - DANE (2013). Cuentas Nacionales por Ramas de actividad económica. Recuperado de: https://www.dane.gov.co/files/.../pib/.../ Ramas_actividad_2012_preli.xls 